\title{
Management of Mirizzi Syndrome in Emergency
}

\author{
Mario Testini, MD, Lucia llaria Sgaramella, MD, ${ }^{1}$ Giuseppe Massimiliano De Luca, MD, PhD, \\ Alessandro Pasculli, MD, Angela Gurrado, MD, PhD, Antonio Biondi, MD, PhD, \\ and Giuseppe Piccinni, MD ${ }^{1}$
}

\begin{abstract}
Introduction: Mirizzi syndrome (MS) is a rare complication of cholelithiasis. Despite the success of laparoscopic cholecystectomy as a minimally invasive approach to gallstone disease, MS remains a challenge, also for open and robotic approaches, due to the subverted anatomy of the hepatocystic triangle. Moreover, when emergency surgery is needed, the optimal preoperative diagnostic assessment could not be always achievable. We aim to analyze our experience of MS treated in emergency and to assess the feasibility of a diagnostic and therapeutic decisional algorithm.

Methods: From March 2006 to February 2016, all patients with a preoperative diagnosis, or an intraoperative evidence of MS, were retrospectively analyzed at our Academic Hospital, including patients operated on in emergency or in deferred urgency. Eighteen patients were included in the study using exclusion criteria and were treated in elective surgery.

Results: The patients were distributed according to modified Csendes' classification: type I in 15 cases, type II in 2, type III in 0 , type IV in 1 , and type V in 0 . In the type I group, diagnosis was intraoperatively performed. Laparoscopic approach was performed with cholecystectomy or subtotal cholecystectomy, when the hepatocystic triangle dissection was hazardous. Patients with preoperative diagnosis of acute abdomen and MS type IV were directly managed by open approach.

Conclusions: Diagnosis of MS and the therapeutic management of MS are still a challenge, mostly in an emergency setting. Waiting for standardized guidelines, we propose a decisional algorithm in emergency, especially in nonspecialized centeres of hepatobiliary surgery.
\end{abstract}

\section{Introduction}

M IRIZZI SYNDROME (MS) is a rare complication of gallstone disease and cholecystitis, characterized by the obstruction of the common hepatic duct by stones impacted at the Hartmann's pouch or cystic duct, showing an incidence approximately less than $1 \%$ of chronic cholelithiaisis in developed Western countries. ${ }^{1}$

After the previous classification by Mc Sherry ${ }^{2}$ and Csendes, ${ }^{3} \mathrm{MS}$ is nowadays graded in five types, as reported by Beltran and Csendes. ${ }^{4}$ Type I includes the evidence of a compression on the common bile duct (CBD) by a stone impacted in the Hartmann's pouch or in the cystic duct; type II, in which appears a cholecystobiliary fistula, results from the erosion of the CBD wall due to a gallstone and affects a third of its circumference; type III involves up to two-thirds of CBD; in type IV, the cholecystobiliary fistula determines a complete destruction of the CBD wall, and the gallbladder is tight with the bile duct forming a single structure with no recognizable dissection planes; and finally, type $\mathrm{V}$ is characterized by a cholecystoenteric fistula associated with an external compression on the CBD or a cholecystobiliary fistula (Fig. 1). This group can also be divided into two subgroups: type Va that includes the association of cholecystoenteric fistula with cholecystobiliary fistula without gallstone ileus, and type $\mathrm{Vb}$ with a cholecystoenteric fistula complicated by gallstone ileus. ${ }^{4}$

Despite the success of laparoscopic cholecystectomy (LC) as a minimally invasive approach to gallstone disease, MS remains a challenge, also for open and robotic approaches ${ }^{5,6}$ due to the subverted anatomy of the hepatocystic triangle. ${ }^{3,5,7,8}$ Moreover, when emergency surgery is needed, the optimal preoperative diagnostic assessment could not be always achievable.

\footnotetext{
${ }^{1}$ Unit of Endocrine, Digestive and Emergency Surgery, Department of Biomedical Sciences and Human Oncology, University Medical School "A. Moro" of Bari, Bari, Italy.

${ }^{2}$ Department of General Surgery, University Medical School of Catania, Catania, Italy.
} 

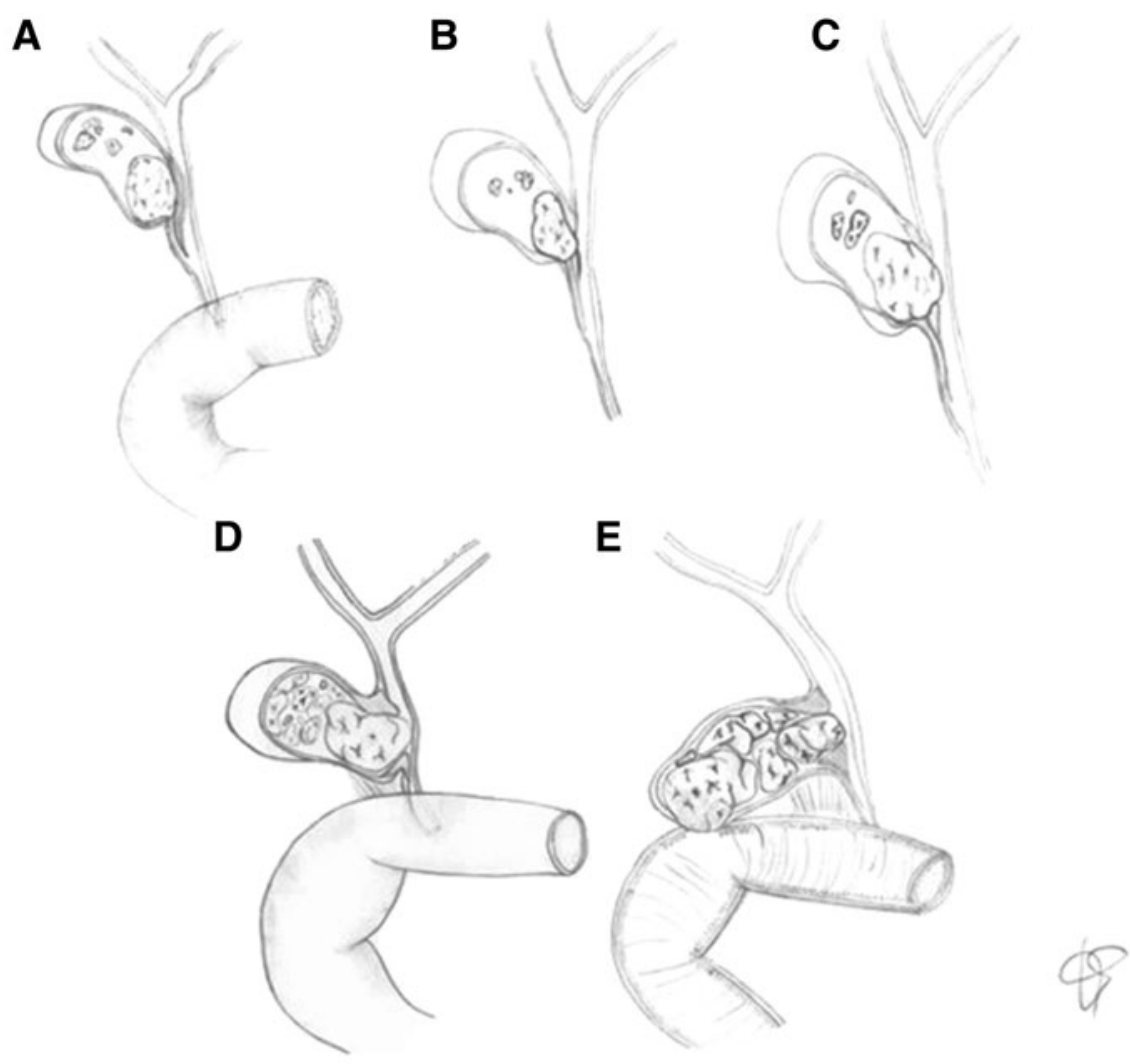

FIG. 1. Mirizzi syndrome, (A) type I, (B) type II, (C) type III, (D) type IV, (E) type V.

Starting from our previous experience regarding MS and laparoscopic iatrogenic lesions, ${ }^{9,10}$ we decided to analyze our data focusing the aim to the emergency setting and to assess the feasibility of a diagnostic and therapeutic decisional algorithm.

\section{Materials and Methods}

From March 1, 2006 to February 29, 2016, a retrospective study was conducted at our Academic Hospital, including patients operated on in emergency or in deferred urgency, in which a preoperative diagnosis or an intraoperative evidence of MS was performed.

Medical records during the study period were evaluated. Demographic data, preoperative diagnosis, type of surgical access and surgical approach, operative time, histology, postoperative complications, and mortality were reordered. Exclusion criteria were represented by patients treated in elective surgery.

Preoperative standard evaluation included routine blood tests comprehending liver function measurements, chest Xray, and electrocardiogram. An ultrasonography of the abdomen was always performed at admission. A multidetector computed tomography or a magnetic resonance cholangiopancreatography (MRCP) was performed when an acute abdomen or an obstructive jaundice was evident, respectively. An endoscopic retrograde cholangio-pancreatography (ERCP) was reserved to patients with obstructive jaundice, cholangitis/pancreatitis.

A written informed consent was always obtained, and during laparoscopic approach, when the dissection of the hepatocystic triangle was not safe due to anatomical subversion, an identification and opening of the gallbladder fundus with subsequent removal of the gallstones in a bag was performed; then, using an inside approach, as described by Hubert, ${ }^{11}$ the Hartmann's pouch was explored with the laparoscope and, starting from the fundus up to the rear portion of the infundibulum, the gallbladder was isolated from the liver with the fundus first technique. The last step was a laparoscopic subtotal cholecystectomy (LSC), applying a linear endoscopic stapler at the Hartmann's pouch. Drainage was left in place in all cases. Conversion to laparotomy was reserved to those cases with dense adhesions to colon, hepatic hilum, and/or duodenum. When the preoperative imaging demonstrated concomitant causes of acute abdomen or an MS type IV, an open approach was directly employed, but when ERCP was immediately available, a nasobiliary drainage (NBD) was placed.

\section{Results}

A total of 919 patients underwent cholecystectomy during the study period. MS was observed in $23(2.5 \%)$ patients and, according to the exclusion criteria, 18 were included in the study.

In $12(66.7 \%)$ patients, a preoperative diagnosis of acute cholecystitis was made. Obstructive jaundice was evident in $5(27.8 \%)$ patients, associated with cholangitis or pancreatitis in one of them $(5.6 \%)$, and with acute cholecystitis in 2 (11.1\%) patients, respectively. In $3(16.7 \%)$ patients with acute abdomen, a CT scan showed an intestinal obstruction 
( $N=2,11,1 \%$ : small bowel volvulus and right colon cancer), or a perforation $(N=1,5,6 \%$ : peptic ulcer), and the diagnosis of MS was intraoperatively and occasionally performed. An MRCP was reserved for 5 patients (27.8\%) with obstructive jaundice, preoperatively revealing a diagnosis of MS. An ERCP with sphincterotomy and clearing of CBD were selectively performed within $24-72$ hours in 5 patients (16.7\%). A total of 15 patients with MS type I were finally detected. When MRCP and ERCP suspected a cholecystobiliary fistula, an NBD was placed $(N=3,60 \%$; type $\mathrm{II}=2$; type $\mathrm{IV}=1)$.

All patients were submitted to emergency surgery in 13 cases $(72.2 \%)$ and to deferred urgent surgery in 5 cases (27.8\%). In 14 patients (77.8\%), a laparoscopic approach was performed, with a conversion rate of $14.3 \%(N=2)$. In 4 patients (16.7\%), an open approach was directly performed due to preoperative diagnosis of intestinal obstruction, perforation, and MS type IV. An LSC was carried out in 4 cases (33.3\%) and an open cholecystectomy was conducted in 4 (22.2\%) with a hepaticojejunal anastomosis, in the case of MS type IV.

Postoperative mortality was $5.6 \%(N=1)$ resulting from multiorgan failure due to severe comorbidities.

Morbidity consisted in two postoperative biliary leakage (13.3\%) in patients with MS type I, managed by ERCP, sphincterotomy, and biliary stenting using a Cotton-Leung prosthesis, removed after 2 months. The follow-up of the remaining 15 patients was uneventful.

Table 1 summarizes the characteristics of patients. In all reported cases, a histological finding compatible with an MS was confirmed, without evidence of malignancy.

\section{Discussion}

The management of MS is controversial and commonly dissimilar in emergency compared with elective surgery. An inexistent literature concerning the approach to MS in emergency justifies the rationale of this study.
Factor considered as predisposing for the development of MS is anatomy, like a long cystic duct parallel to the CBD, or its low insertion site. ${ }^{3,4}$ The impact of gallstones determines a chronic inflammation of the gallbladder wall and of the cystic duct, leading to the involvement of the CBD in phlogistic process, with a close adhesion of gallbladder and choledocus, leading to cholecystobiliary or cholecystoduodenal fistula.

Diagnosis of MS is still a challenge, mostly in an emergency setting. Indeed, from 1995 to 1999 , Schafer ${ }^{12}$ analyzed 13,000 LCs and found 39 cases of MS, classified into 34 MS type I and 5 MS type II according to Mc Sherry, and MS was preoperatively assumed only in 18 cases $(46.1 \%)$.

Indeed, although obstructive jaundice is the most common clinical presentation, ${ }^{13}$ already directing the diagnosis toward more complete imaging in emergency, sometimes this sign could not be yet apparent, as in these series $(27.8 \%)$. However, acute cholecystitis with pain and fever has proved to be the prevailing clinical feature $(66.7 \%),{ }^{13}$ often limiting the preoperative imaging to ultrasonography, and moving toward an emergency operation. For this reason, these patients are usually managed in not-dedicated centeres of hepatobiliary pancreatic surgery, with the risk of severe sequelae, potentially up to liver transplantation. In effect, the hepatobiliary ultrasonography shows limited sensitivity, varying from $8.3 \%$ to $27 \% .{ }^{14}$ It can reveal generic and indirect information related to a suspected MS as a contracted gallbladder, with thick or extremely thin walls, containing a single large gallstone or multiple smaller gallstones impacted in the infundibulum. Moreover, the biliary tract would be dilated in its extra and/or intrahepatic portions upstream of the obstruction, but with a normal caliber of the distal portion.

CT scan shows dilation of the intrahepatic duct with the presence of stones in the biliary tree; however, the evidence of periductal inflammation may be misinterpreted as gallbladder cancer. In our experience, the use of this technique was reserved to patients with acute abdomen, in which the MS was an occasional intraoperative finding.

Table 1. Characteristics of Patients

\begin{tabular}{|c|c|c|c|c|c|}
\hline & $\begin{array}{l}\text { MS type I } \\
(\mathrm{N}=15)\end{array}$ & $\begin{array}{l}\text { MS type II } \\
\quad(\mathrm{N}=2)\end{array}$ & $\begin{array}{c}\text { MS type III } \\
(\mathrm{N}=0)\end{array}$ & $\begin{array}{c}\text { MS type IV } \\
(\mathrm{N}=1)\end{array}$ & Total $(\mathrm{N}=18)$ \\
\hline Mean age (years) & $62.4 \pm 11.3$ & $61 \pm 9.9$ & - & $60 \pm 0$ & $62.1 \pm 10.5$ \\
\hline Sex F/M & $9 \mathrm{~F} / 6 \mathrm{M}$ & $2 \mathrm{~F} / 0 \mathrm{M}$ & - & $1 \mathrm{~F} / 0 \mathrm{M}$ & $12 \mathrm{~F} / 6 \mathrm{M}$ \\
\hline $\begin{array}{l}\text { Clinical evidence } \\
\text { of cholecystitis }\end{array}$ & $12(80.0)$ & $0(0)$ & - & $0(0)$ & $12(66.7)$ \\
\hline Obstructive jaundice & $2(13.3)$ & $2(100)$ & - & $1(100)$ & $5(27.8)$ \\
\hline Acute abdomen & $3(20.0)$ & $0(0)$ & - & $0(0)$ & $3(16.7)$ \\
\hline Preoperative diagnosis & $0(0)$ & $2(100)$ & - & $1(100)$ & $3(16.7)$ \\
\hline Laparoscopic approach & $12(80.0)$ & $2(100)$ & - & $0(0)$ & $14(77.8)$ \\
\hline $\begin{array}{l}\text { Laparoscopic } \\
\text { cholecystectomy }\end{array}$ & $8(53.3)$ & $0(0)$ & - & $0(0)$ & $8(44.4)$ \\
\hline $\begin{array}{l}\text { Laparoscopic subtotal } \\
\text { cholecystectomy }\end{array}$ & $2(13.3)$ & $2(100)$ & - & $0(0)$ & $4(33.3)$ \\
\hline Open approach & $3(20.0)$ & $0(0)$ & - & $1(100)$ & $4(16.7)$ \\
\hline Conversion rate & $2(16.7)$ & $0(0)$ & - & $0(0)$ & $2(14.3)$ \\
\hline Operative time (minutes) & $129.7 \pm 39.2$ & $145 \pm 7.1$ & - & $220 \pm 0$ & $136.4 \pm 41.5$ \\
\hline Postoperative complication & $\begin{array}{l}2 \text { biliary } \\
\text { leakage (13.3) }\end{array}$ & $0(0)$ & - & $0(0)$ & $\begin{array}{l}2 \text { biliary } \\
\text { leakage (11.1) }\end{array}$ \\
\hline Mortality & $1(6.6)$ & $0(0)$ & - & $0(0)$ & $1(5.6)$ \\
\hline
\end{tabular}

Data are given as mean value and standard deviation or as absolute value and percentage.

MS, Mirizzi syndrome. 
MRCP is the gold standard examination in the preoperative imaging of $\mathrm{MS}$, demonstrating high specificity and sensitivity with an accuracy of more than $90 \%$, detecting gallstones and bile duct stenosis. It can also show the extrinsic compression of the bile duct and it determines whether a fistula is present, differentiating between MS and other causes of bile tract obstruction. ${ }^{15}$ But, this imaging technique is usually reserved to patients with obstructive jaundice, being too much expensive for all patients with gallstone disease. Moreover, not all radiological departments have it in emergency.

ERCP is an invasive procedure with diagnostic and therapeutic finality and shows an accuracy of around 55-99\%. The correct diagnosis of MS before any surgical procedure is of extreme importance to avoid CBD injuries. Even if in our series and in our algorithm ERCP may appear overused
$(16.7 \%)$, we believe that it helps not only in identifying cholecystobiliary or cholecystoenteric fistulae (such as MRCP, performed in patients with jaundice) but also in quantifying their importance, allowing to leave an NBD and to plan the best surgical approach, up to biliary-digestive reconstruction.

The surgical management of MS depends on the type of MS, the degree of biliary inflammation, and on the hepatobiliary training of surgeons. ${ }^{16}$ The anatomopathological aspect of the biliary tract poses the question of the appropriateness of LC, because of the potential increased risk of iatrogenic lesion of CBD. ${ }^{17,18}$ Dealing with this, when anatomy is not clear, and/or surgical dissection and performability of Strasberg maneuver is extremely difficult or hazardous, a conversion to open surgery should be mandatory. However, a subtotal cholecystectomy both in laparoscopic or in open

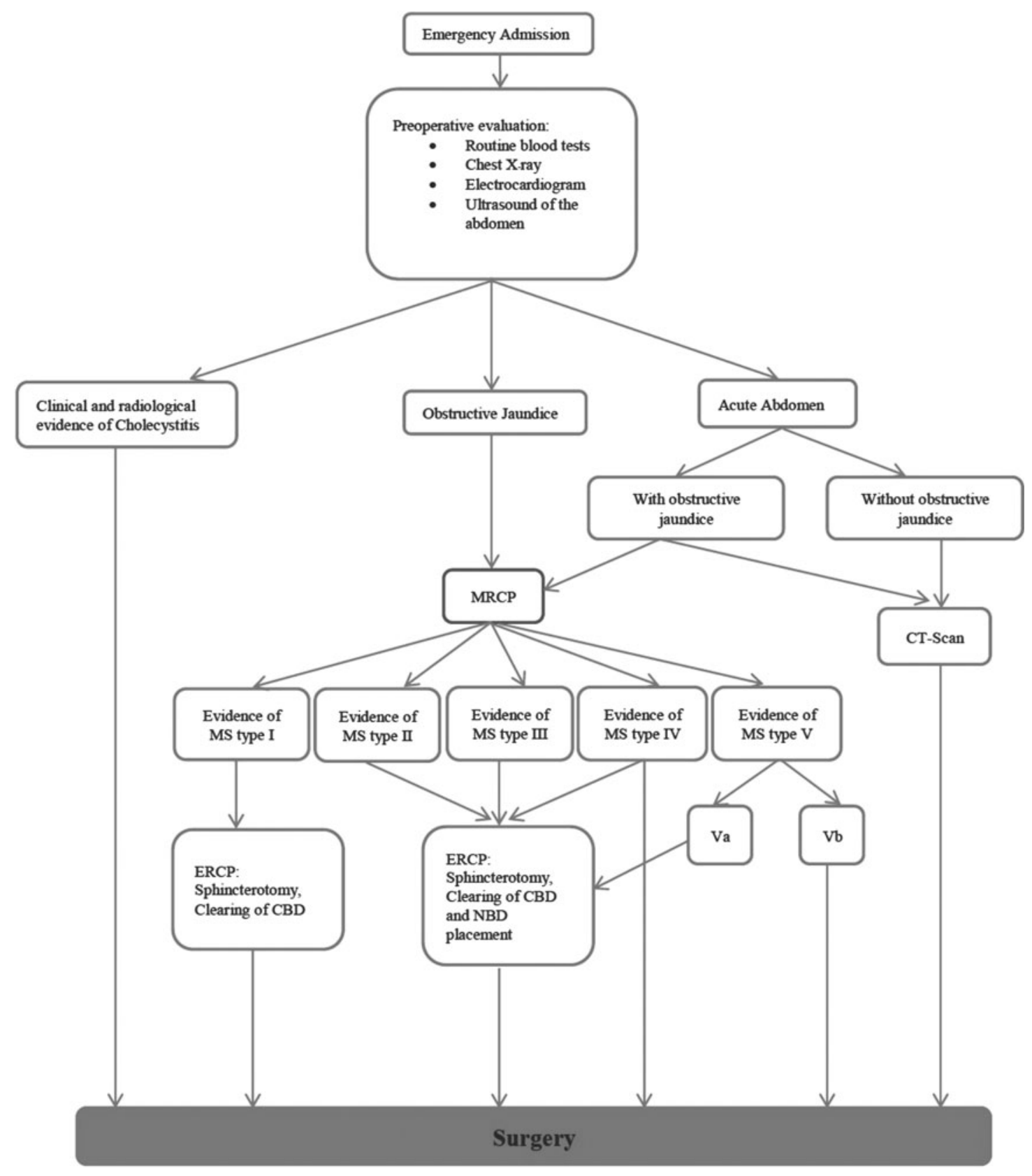

FIG. 2. Decisional algorithm summarizing management of MS. MS, Mirizzi syndrome. 
approaches is considered as the suitable treatment for some MS type I and in most cases of MS types II and III, ${ }^{16,18,19}$ avoiding higher risk of CBD and duodenal injuries secondary to $\mathrm{LC}^{10}$ compared with a low risk of postoperative complication (residual gallstone and biliary fistula). In our experience, this intraoperative strategy was useful in $22.2 \%$, without complications during the follow-up. From 2003 to 2005 , Sinha et al. ${ }^{20}$ performed 889 laparoscopic cholecystectomies, with the aim of establishing an alternative to conversion to open surgery in complicated cases, and in $28(3.1 \%)$ cases they carried out an LSC, without the dissection of hepatocystic triangle and without ligation of the cystic duct, reducing the conversion rate to $0.3 \%$. Type IV should be managed in two ways, normally in open approach. ${ }^{17}$ When the CBD defect is small, a termino-terminal (T-T) anastomosis of the residual stumps of the CBD or a reconstruction of the biliary tract wall using the remaining part of gallbladder could be employed, using a T-tube in both cases. When the defect is large, a biliarydigestive anastomosis should be performed. ${ }^{17}$

Recent studies even proposed the robotic approach with the Da Vinci Surgical System (Intuitive Surgical Mountain View, $C A$ ) as an alternative to laparoscopy in selected patients, ${ }^{21}$ but the still small sample of patients is nowadays a limit to consider this approach as routine.

This study is limited by its retrospective design and the relatively few patients. Nonetheless, it could contribute to propose a decisional algorithm (Fig. 2) in the management of patients affected by MS in emergency, sometimes in nonspecialized centers of hepatobiliary surgery. At least in doubtful cases, a more widespread preoperative MRCP could be very useful, also in emergency, reducing intraoperative accidents and moving patients at risk of MS to dedicated centers.

In conclusion, the results of this study confirm that subtotal cholecystectomy can be a way out of MS types II and III, and of type I in which the intraoperative framework is not clear. The open or laparoscopic management should depend on the specific skill of the surgeon. Conversely, types IV and V should always be approached by laparotomy.

Prospective studies are needed to standardize guidelines.

\section{Acknowledgment}

The authors are grateful to Dr. Claudia Ventrelli for writing assistance and review of the article in English.

\section{Disclosure Statement}

No competing financial interest exists.

\section{References}

1. Abou-Saif A, Al-Kawas FH. Complications of gallstone disease: Mirizzi syndrome, cholecystocholedochal fistula, and gallstone ileus. Am J Gastroenterol 2002;97:249-254.

2. Mc Sherry CK, Ferstenberg H, Virshup M. The Mirizzi syndrome: Suggested classification and surgical treatment. Surg Gastroenterol 1982;1:219-225.

3. Csendes A, Diaz JC, Burdiles P, Malueda F, et al. Mirizzi syndrome and cholecystobiliary fistula: A unifying classification. Br J Surg 1989;76:1139-1143.

4. Beltran MA, Csendes A, Cruces KS. The relationship of Mirizzi syndrome and cholecystoenteric fistula: Validation of a modified classification. World J Surg 2008;32:22372243.
5. Antoniou SA, Antoniou GA, Makridis C. Laparoscopic treatment of Mirizzi syndrome: A systematic review. Surg Endosc 2010;24:33-39.

6. Mithani R, Schwesinger WH, Bingener J, et al. The Mirizzi syndrome: Multidisciplinary management promotes optimal outcomes. J Gastrointest Surg 2008;12:1022-1028.

7. Kok KY, Goh PY, Ngoi SS. Management of Mirizzi's syndrome in the laparoscopic era. Surg Endosc 1998;12:1242-1244.

8. Martin RF, Rossi RL. Bile duct injuries. Spectrum, mechanisms of injury, and their prevention. Surg Clin North Am 1994;74:781-803; discussion 805-807.

9. Piccinni G, Sciusco A, De Luca GM, Gurrado A, Pasculli A, Testini M. The minimanllyt invasive treatment of mirizzi's syndrome: There a safe way? Ann Hepatol 2014;13: 558-564.

10. Testini M, Piccinni G, Lissidini G, et al. Management of descending duodenal injuries secondary to laparoscopic cholecystectomy. Dig Surg 2008;25:12-15.

11. Hubert C, Annet L, Van Beers BE, et al. The "inside approach of the gallbladder" is an alternative to the classic Calot's triangle dissection for a safe operation in severe cholecystitis. Surg Endosc 2010;24:2626-2632.

12. Schafer M, Schneiter R, Krahenbuhl L. Incidence and management of Mirizzi syndrome during laparoscopic cholecystectomy. Surg Endosc 2003;17:1186-1190; discussion 1191-1192.

13. Zhong H, Gong JP. Mirizzi syndrome: Experience in diagnosis and treatment of 25 cases. Am Surg 2012;78:61-65.

14. Al-Akeely MH, Alam MK, Bismar HA, et al. Mirizzi syndrome: Ten years experience from a teaching hospital in Riyad. World J Surg 2005;29:1687-1692.

15. Safioleas M, Stamatakos M, Safioleas P, et al. Mirizzi syndrome: An unexpected problem of cholelithiasis. Our experience with 27 cases. Int Semin Surg Oncol 2008;5:12.

16. Beltran MA. Mirizzi syndrome: History, current knowledge and proposal of a simplified classification. World J Gastroenterol 2012;18:4639-4650.

17. Baer HU, Matthews JB, Schweizer WP, et al. Management of the Mirizzi syndrome and the surgical implications of cholecystcholedochal fistula. Br J Surg 1990;77:743-745.

18. Sandblom P, Tabrizian M, Rigo M, et al. Repair of common bile duct defects using gallbladder or cystic duct as a pedicled graft. Surg Gynecol Obstet 1975;140:425-432.

19. Ransom KJ. Laparoscopic management of acute cholecystitis with subtotal cholecystocholedochal fistula. Am Surg 1998;64:955-957.

20. Sinha I, Smith ML, Safranek P, et al. Laparoscopic subtotal cholecystectomy without cystic duct ligation. Br J Surg 2007;94:1527-1529.

21. Tung KL, Tang CN, Lai EC, et al. Robot-assisted laparoscopic approach of management for Mirizzi syndrome. Surg Laparosc Endosc Percutan Tech 2013;23:e17-e21.

Address correspondence to: Mario Testini, $M D$ Unit of Endocrine Digestive and Emergency Surgery Department of Biomedical Sciences and Human Oncology University Medical School “A. Moro” of Bari Policlinico, Piazza Giulio Cesare, 11 Bari 70124 Italy

E-mail: mario.testini@uniba.it 